\title{
Debats autour de la norme et de la qualite de la langue en milieu minoritaire : l'exemple de l'Acadie du Nouveau-Brunswick ${ }^{1}$
}

\author{
Laurence Arrighi ${ }^{2}$ \\ Émilie Urbain ${ }^{3}$
}

Résumé: En nous appuyant sur les discours publiés dans les principaux médias francophones de la région ces cinq dernières années, nous examinons les discours sur la langue française en circulation dans une communauté francophone minoritaire du Canada, l'Acadie du Nouveau-Brunswick. Lobjectif de notre article est de dresser un portrait des débats sur la qualité du français et la norme linguistique qui émergent dans cette région caractérisée par l'usage répandu de vernaculaires marqués par la variation linguistique (que ce soit en termes d'archaïsmes, de variation morphosyntaxique ou encore de contact des langues). Dans un contexte minoritaire où les contacts linguistiques avec la langue dominante, l'anglais, sont nombreux et multiformes, les discours sur la qualité du français parlé (ou écrit) dans la région sont politiquement chargés et s'articulent à des questions de nationalisme, de représentation politique et de droit. Notre étude montre ainsi comment les propos sur la langue, qu'ils soient portés par des acteurs sociaux ordinaires ou par des personnalités publiques, donnent à voir des enjeux sociaux, politiques et économiques plus larges. En particulier, nos analyses révèlent que les discours souvent critiques sur le français des Acadiens et des Acadiennes s'articulent fréquemment autour de questions d'accès et de partage des ressources: accès au marché de l'emploi, accès à des services publics, accès à la francophonie internationale et les opportunités de développement économique quelle pourrait permettre.

Mots clés: Français acadien. Langue standard. Minorités linguistiques. Canada

\section{T 'objectif de cette contribution est d'offrir un aperçu des débats sur la qualité du français et la norme linguistique tels qu'ils se}

\footnotetext{
1 Le point de départ de ce travail émane d'une recherche menée initialement en 2018 afin d'offrir un bref portrait des débats sur la norme linguistique en Acadie dans un ouvrage à destination du grand public (à paraitre). Outre la mise à jour des données analysées, la présente étude offre une contextualisation historique et conceptuelle de ces discours métalinguistiques.
}

2 Université de Moncton.

3 Université de Carleton. 
donnent à voir dans les médias d'une communauté francophone minoritaire canadienne, celle de l'Acadie du Nouveau-Brunswick.

Dans un premier temps, nous brosserons un rapide portrait sociolinguistique de cette communauté destiné à donner aux lecteurs et lectrices un aperçu de la francophonie acadienne. Cet aperçu vise à permettre une meilleure compréhension des idéologies linguistiques en circulation en Acadie. Ces idéologies seront saisies dans notre partie analytique comme l'un des fondements explicatifs des rapports ambivalents aux questions de norme et de qualité de la langue au sein de la communauté étudiée. Dans un deuxième temps, en partant d'un corpus de discours médiatique des cinq dernières années, nous proposons une analyse des débats sur la norme et la qualité de la langue en Acadie en mettant principalement de lavant les thèmes particulièrement débattus et les arguments avancés. Nous replacerons, ce faisant, ces débats dans le contexte de la longue histoire documentée des idéologies linguistiques en Acadie et plus largement au Canada francophone. Avec des exemples récents, nous montrerons que, quel que soit lévénement d’actualité déclencheur, ces débats sont prétextes à un discours où idéologies de la langue et idéologies de la nation s'entremêlent.

\section{Portrait sociolinguistique de la francophonie néo-brunswickoise}

La situation de francophonie minoritaire vécues par les Acadiens et Acadiennes du Nouveau-Brunswick explique, en bonne partie, un rapport aux questions de langue que l'on pourrait qualifier, à la suite de Bouchard (2002) d'obsessionnel. Les travaux de Boudreau sur l'Acadie du NouveauBrunswick $(2009,2016)$ ainsi que d'Arrighi et Violette (2013) et d'Arrighi et Urbain $(2016,2016 / 2017,2019)$ étudient, à partir de données empiriques, les représentations linguistiques que tiennent les locuteurs et locutrices à l'égard du français parlé en Acadie. Si les pratiques régionales sont 
largement évaluées de manière défavorable, il arrive que certaines d’entre elles, soient, selon leur origine, investies de certaines valeurs positives. Ces représentations sont largement héritières de l'histoire ainsi que de la situation sociolinguistique de la communauté.

La présence historique d'une communauté de langue française au nord-est de l'Amérique du Nord, située sur les bords del'Atlantique et séparée de la communauté québécoise bien plus connue et démographiquement plus importante, est liée à la colonisation française de l'Amérique qui débuta en 1604 par la fondation le long de la Baie de Fundy de la colonie d'Acadie (celle dite du Canada, aujourd'hui Québec, voit le jour quatre années plus tard). Dès 1713, les aléas de l'histoire vont que la France perd définitivement l'Acadie. La colonie passe en des mains anglaises, mains qui en 1755 organisent la déportation de la population dorigine française (DAIGLE, 1993). Cet événement connu sous le nom de Grand dérangement explique l'effondrement numérique des descendants de colons français, leur dispersion sur un vaste territoire le long de la façade atlantique et in fine la fragilisation du groupe acadien. Le fait français ne disparait pas pour autant de cette région du monde et, dès le milieu du $19^{\mathrm{e}}$ siècle, les francophones de ce dominion britannique travaillent à obtenir droit et reconnaissance. À la fin du $19^{\mathrm{e}}$ siècle, ils sont suffisamment puissants pour se doter d'institutions (établissements d’enseignement supérieur, journaux, caisses mutuelles d'entraide) et d'insignes nationales (un drapeau, un hymne, une fête nationale etc.) (BOURQUE ; RICHARD, 2014). Toutefois, il faudra attendre encore plus d'un siècle pour que les législations fédérale et provinciale reconnaissent des droits à cette communauté linguistique.

Ladoption de la loi fédérale sur les langues officielles en 1969 apporte de réelles avancées au niveau légal en ce qui concerne les compétences et services dépendant du gouvernement fédéral. Le Nouveau-Brunswick est la seule province à avoir adopté un modèle de bilinguisme officiel au niveau provincial. Concrètement, cela veut dire que la population peut être 
servie par l'État dans la langue officielle de son choix. En éducation, santé et services communautaires sapplique un principe de dualité linguistique qui signifie qu'une institution (école, hôpital, etc) est gérée par une des communautés de langue officielle. Pour plusieurs experts, l'épanouissement des communautés francophones minoritaires canadiennes se jouerait sur leur capacité à préserver, à développer et à contrôler des espaces institutionnels unilingues afin doffrir des occasions de vivre pleinement en français. Pour paraphraser le titre d'un travail récent (FORGUES et al., 2020), la construction d'espaces unilingues serait ainsi un véritable projet de société dans la francophonie canadienne.

Si les défis restent toujours nombreux pour ces francophones en milieu minoritaire, la communauté acadienne du Nouveau-Brunswick dispose néanmoins de plusieurs atouts. Statistiquement, les Acadiens et les Acadiennes représentent plus d'un tiers des habitants de la province (rappelons que dans nulle autre province canadienne, hormis la province de Québec, les francophones ne dépassent les 5\%). Le poids du nombre apporte non seulement une force politique notable, mais cette masse critique de locuteurs et locutrices du français permet aussi, au quotidien, la pratique de sa langue en de multiples situations.

Certaines régions acadiennes de la province comme le nordest (région dite de la Péninsule acadienne) ou le nord-ouest (région du Madawaska) sont presque à 100\% francophones et les gens y vivent pleinement en français. La région du sud-est en revanche n'est francophone quà un tiers. La fréquentation quotidienne des locuteurs de l'autre langue officielle, l'anglais (langue bien plus forte à l'échelle de tout le continent) entraine nécessairement des effets linguistiques de contact.

Or, dans la mesure où la pureté linguistique, en Acadie comme ailleurs, est le modèle idéal type de la « bonne langue » (ROBILLARD, 2001 ; KLINKENBERG, 2001 ; HELLER, 2011; PAVEAU ; ROSIER, 2008 ; CANDEA ; VERON, 2019), l'existence de traces linguistiques du contact 
français-anglais constitue "un terrain idéologique miné » (LECLERC, 2016, p. 96) et ce de longue date (BOUCHARD, 2002, 2011 ; BOUDREAU, 2009), comme nous aurons l'occasion de le voir.

L'une des conséquences de cette disparité entre les régions en termes de contact avec l'anglais est que les locuteurs et locutrices du sud-est sont souvent montrés du doigt alors que ceux et celles du nord peuvent être brandis comme modèles.

Pour autant, la situation des Acadiens et Acadiennes du Sud-est présente aussi quelques avantages non négligeables. C'est au Sud-Est que se trouve le principal campus de l'Université de Moncton, plus grande institution d'enseignement supérieur en français hors du Québec. C’est encore cette région qui offre les meilleures possibilités d’emploi à haute valeur ajoutée aux francophones (aux francophones bilingues), dans la fonction publique fédérale ou provinciale, dans le domaine de la santé, de léducation, dans le monde associatif, dans le secteur culturel, dans les télécommunications etc. Cette région attire de plus en plus les francophones du reste du Nouveau-Brunswick (et d'ailleurs dans le monde, VIOLETTE, 2010). Le français qui s'y parle, parfois subsumé sous le nom de chiac, est un français marqué par le mélange des langues et /ou la présence de mots et locutions héritées d'anciennes formes du français (PERROT, 1995 ; ARRIGHI ; BERGER, à paraitre). C’est particulièrement cette variété de langue qui fait objet de débats dans la mesure où la présence d’emprunts à l’anglais éloigne ce français de la norme. Ce débat est aussi récurrent que virulent comme nous allons le voir.

\section{Prises de position sur la qualité de la langue dans les médias}

La qualité du français parlé par les Acadiens et les Acadiennes et le rapport à la norme linguistique de ces derniers sont des sujets qui agitent régulièrement l'espace public et médiatique régional (BÉRUBÉ, 
2010; BOUDREAU, 2016) voire national (ARRIGHI; VIOLETTE, 2013 ; ARRIGHI; URBAIN, 2016/2017, 2019) et ce depuis les débuts de la presse acadienne (BOUDREAU, 2009). Comme ailleurs dans la francophonie ${ }^{4}$, lorsque le français régional est comparé avec d’autres variétés de français, notamment le français de France, cela amène certains à questionner la légitimité des particularités linguistiques acadiennes. De telles positions ont déjà été mises de l'avant pour d'autres terrains (en Belgique par Francard, 1993; en France par Gueunier, Genouvrier et Khomsi,1978; Ledegen, 2000 notamment). Le plus souvent, la comparaison saccompagne d'une évaluation de la qualité de la langue française défavorable aux «périphéries » (FRANCARD, 2018).

Les régionalismes ne sont cependant pas toujours vus d'un mauvais œil. Des prononciations, du vocabulaire, des structures syntaxiques peuvent être investis de valeurs tantôt positives, lorsque ces pratiques linguistiques sont associées à l'identité collective, et tantôt négatives lorsquelles sont vues comme la manifestation de l'imminence de l'assimilation linguistique ou encore comme preuve d'un faible niveau de littératie ou d'un manque de volonté et de fierté. La culpabilisation des individus accusés de ne pas en faire assez pour leur langue est l'un des grands motifs discursifs des discours sur la norme et la qualité de la langue (ARRIGHI ; VIOLETTE, 2013 ; ARRIGHI ; URBAIN, 2016 ; KLINKENBERG, 2001).

Afin de saisir les arguments mis de l'avant et les enjeux soulevés par ce discours sur notre terrain, nous avons analysé les débats sur la qualité de la langue des Acadiens et des Acadiennes dans les principaux médias francophones néo-brunswickois sur les cinq dernières années.

4 Pour d'autres régions canadiennes, voir MONNIN; HALLION, 2016/2017; BOUCHARD, 2002 et 2011; REMYSEN, 2012. 
Nos analyses portent plus précisément sur les discours ayant circulé dans le quotidien L'Acadie Nouvelle ${ }^{5}$ et sur le site web de Ici Radio-Canada

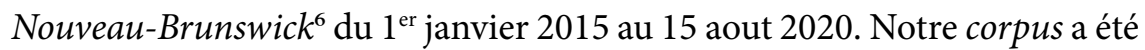
constitué à l'aide de la base de données Eureka, à partir de trois recherches systématiques par mots-clés (qualité + langue; chiac; français acadien).

Les questions de langue sont un thème récurrent dans les médias acadiens. Pour le présent propos, nous avons sélectionné, parmi les résultats, les textes proposant des commentaires évaluatifs sur les pratiques linguistiques des Acadiens et Acadiennes. Ainsi, nous avons laissé de côté un certain nombre d'articles traitant de la qualité du français des anglophones: que ce soit sur les compétences des élèves qui sortent des programmes d'immersion, sur des commerçants incapables d’offrir un service dans la langue de la clientèle francophone ou encore, tout au long du printemps et de l'été 2020, sur le fait que des politiciens néo-brunswickois peinaient à s'adresser en français aux citoyens et citoyennes lors des misesà-jour gouvernementales sur la crise sanitaire de la Covid-19. Nous avons également exclu pour cette analyse les textes traitant de la qualité des services dans la langue minoritaire officielle, comme par exemple la polémique sur le manque de services en français chez Ambulances Nouveau-Brunswick ${ }^{7}$.

Le corpus retenu et analysé est constitué de 231 textes. Ce sont des articles, des chroniques, des reportages et un certain nombre de lettres d'opinion. Ce sont les textes d'opinion qui offrent souvent les argumentaires les plus développés et détaillés sur la question de la langue.

Le choix de saisir un corpus médiatique se justifie par le rôle de

5 Le journal L'Acadie Nouvelle dessert quotidiennement la population francophone du Nouveau-Brunswick depuis 1984, avant ce titre de presse deux autres journaux ont pourvu la communauté en médias quotidiens dans sa langue et ce depuis la fin du $19^{\mathrm{e}}$ siècle.

6 Le radiodiffuseur et télédiffuseur national émet des émissions préparées localement à partir de Moncton. Une partie de ce qui se trouve sur le site du diffuseur est donc de conception régionale, le reste vient de Montréal.

7 Auparavant offerts par les municipalités, les services médicaux d'urgence, sont depuis une dizaine d'années centralisés par Ambulances NB. Cette société fait depuis plusieurs années et de façon récurrente face à des critiques de la part du public et à des plaintes légales formelles quant à l'application de la loi provinciale sur les langues officielles. 
premier plan joué par la presse (et désormais d’autres médias, dont les médias en ligne) dans la formulation et la diffusion des représentations et des idéologies linguistiques (KROSKRITY, 2000 ; JOHNSON; MILANI, 2010; URBAIN, 2014; au Canada français : HELLER, 2010 ; BOUDREAU, 2009; BOUDREAU; URBAIN, 2013).

Au Canada, dès la deuxième moitié du $19^{\mathrm{e}}$ siècle, lélite canadiennefrançaise s'y "positionne [...] par rapport aux usages de l’époque, que ce soit les archaïsmes, les régionalismes ou les anglicismes. " (CROTEAU; MARTINEAU; FRENETTE, 2018, p. 173). En parallèle ajoutent ces chercheurs, "la presse a constitué un des principaux canaux de formulation, de promotion et de diffusion d'un projet de construction nationale fondé sur la langue française " (CROTEAU; MARTINEAU ; FRENETTE, 2018, p. 174).

On comprend dès lors que lorsque, en Acadie, on parle de la langue, de sa maitrise, de sa conformité, de sa qualité, les enjeux sont immenses.

Comme le montrent de nombreux travaux s'inscrivant en sociolinguistique critique (HELLER, 2002; CAMERON, 1997), les débats langagiers renferment souvent des dimensions idéologiques et politiques. Ainsi, quand on parle de conformité et de pureté linguistiques ainsi que de qualité de la langue, les prises sont loin dêtre neutres : à travers la langue, on parle d'autre chose. Dans notre corpus, on ne parle pas seulement de l'existence de différentes façons de parler, mais de toute une série d'enjeux qui y sont associés: la qualité du système éducatif de la province, les défis de la mondialisation, la place des francophones sur le marché de l'emploi, la préservation de l'identité acadienne, les responsabilités citoyennes envers la collectivité, l'avenir de la francophonie canadienne, la vitalité linguistique de la communauté etc. La communauté acadienne se définit, outre par son histoire et des valeurs communes, par le partage d'une langue, reprenant en cela les grands poncifs du modèle de construction des État-nations (HELLER, 2007, 2011). On comprend dès lors que les débats remettant en cause la qualité même de cette langue partagée dépassent les simples enjeux 
formels et soient à ce point investis émotionnellement par les acteurs sociaux qui y participent.

Ces débats surgissent fréquemment à loccasion de toute une variété d'événements déclencheurs. Par exemple, en aout 2019, L'Acadie Nouvelle publie une série de lettres du lectorat aux titres évocateurs : "Surdose de franglais " (Mon opinion, Pierre LeBlanc, L’Acadie Nouvelle, 23 aout 2019, "L'Acadie mérite mieux » (Mon opinion, Benoît Duguay, L'Acadie Nouvelle, 30 aout 2019) en réaction à des spectacles du 15 aout (jour de la fête nationale de l’Acadie) jugés trop anglicisés.

On retrouve dans la presse nombre d'exemples d'événements qui ont mis le feu aux poudres: rencontres sur la politique d'aménagement linguistique du système scolaire francophone de la province, taux d'échec d'étudiants et étudiantes de la Faculté d'éducation de l'Université de Moncton à un test de maitrise des compétences linguistiques, échec de la clientèle étudiante en soins infirmiers de la même institution à un test provincial d'habilitation professionnelle, choix d'un slogan chiac, " right fier », pour les Jeux de la francophonie canadienne organisés à Moncton et Dieppe en 2017, commentaires de personnalités publiques (par exemple le chroniqueur du journal montréalais Le Devoir, Christian Rioux ou l'essayiste Denise Bombardier) etc.

Il est très fréquent que les débats aient comme point de départ des rencontres entre la francophonie acadienne et d'autres communautés francophones canadiennes ou internationales, comme lors des Jeux de la francophonie, par exemple. La comparaison du français acadien avec des variétés plus légitimes amène de nombreux scripteurs de notre corpus à condamner les différences linguistiques et à valoriser la norme exogène.

Souvent aussi, des commentateurs se veulent des lanceurs d'alerte face à une société civile acadienne qu'ils jugent trop complaisante voire apathique. Citons presque intégralement une lettre de lecteur et un extrait d'une chronique : 
Permettez-nous de revenir sur la question de «l'insécurité linguistique»

[...] belle [..] idée [...] : «L'insécurité linguistique», on devrait plutôt se préoccuper de «linconscience linguistique» parce que celle-ci fait bien plus de ravages que l'autre dans notre langue et notre culture acadienne. Exemples de cette inconscience, entre autres, le slogan «right fier» et les garderies bilingues. En donnant au chiac des lettres de noblesse, sous prétexte de ne pas brimer les jeunes dans leur façon de s'exprimer, on nuit à ces mêmes jeunes face au marché du travail et à la mondialisation. Les écoles ont un rôle majeur dans la promotion de la langue et de la culture et le personnel enseignant devrait se montrer plus exigeant dans ce contexte. [...] (DUGUAY, 2018)

[...] jai été littéralement stu-pé-fait d'entendre certaines têtes d'affiche de l'Acadie la plus classique qui soit s'esbaudir, avec jubilation appuyée, du fait que de jeunes francophones choisissent d'exprimer la quintessence de leur propre «fierté française» via le désormais notoire slogan «right fiers» moitié anglais moitié-chiac, qui cristallise en deux mots l'ampleur de la déréliction linguistique d'une génération happée par les mirages d'une lingua franca qui, pourtant, se contrefiche de l'Acadie, de son histoire, sa réalité, sa culture et ses aspirations. Et même de son existence.

Il en est de notre langue et de notre culture comme des plantes: même si lon ne voit pas toujours leurs racines profondes ou lointaines, il demeure essentiel de les entretenir dans le meilleur terreau si l'on veut que cette langue et cette culture puissent s'épanouir et rayonner. 
À moins de vouloir faire croire au monde qu'une plante en plastique peut donner de belles fleurs parfumées. Ciel! Assimilons-nous au plus vite: on n'aura plus à faire semblant. (ROSSIGNOL, 2016)

À l'instar de ces exemples, un but implicite des articles condamnant la qualité de la langue en Acadie est de faire réagir la communauté. Cette exhorte vaut alors pour les médias, le monde politique, associatif et surtout les jeunes, les parents et les éducateurs. Le devoir est collectif comme l'indiquent les deux extraits ci-dessous :

Le parent fier et qui tente d'employer en tout temps un français correct transmet à son enfant une valeur qui s'enracine. L'enseignant ou l'enseignante, qu'il soit professeur de langue, de sciences ou d'histoire, de première ou de douzième année, exerce une influence tout aussi grande. Si on pouvait en être tous un peu plus conscients, on changerait le monde. On rendrait un énorme service aux jeunes en les outillant de tout un bagage qui permet dêtre plus performants dans toutes les formes de la communication. (CORMIER, 2018)

Les questions de fond concernant l'usage et la qualité du français ne sont pas réservées à quelques spécialistes, mais nous concernent tous. Le français mérite nos petits soins. Le français n'est pas une langue comme les autres. C'est la langue dans laquelle nous, les francophones, avons appris à parler. (RENAULT, 2018)

Si autant de scripteurs enjoignent la communauté à surveiller sa langue, c'est que cette dernière est tenue pour menacée, en danger et ce, avant tout, dans sa forme même. Les idées que nous trouvons développées à propos de la qualité de la langue tout au long de notre corpus sont les 
mêmes que celles dont on trouve traces depuis la fin du $19^{\mathrm{e}}$ siècle dans la presse acadienne (BOUDREAU, 2009, 2014) et plus largement canadiennefrançaise (BOUCHARD, 2002). Dans l'ensemble, lorsque l’on parle de norme linguistique c'est pour souligner les écarts par rapport à celle-ci et dénoncer la piètre qualité de la langue. Ce sont le plus souvent les emprunts linguistiques (et les autres types d'anglicismes) et plus largement le mélange des langues qui sont sévèrement critiqués. Un lecteur de L’Acadie Nouvelle propose une véritable saynète dans laquelle il se met en scène en client outragé par l'emploi d'un calque de l'anglais:

Je suis francophone. J'habite un lieu où au moins $95 \%$ des habitants sont francophones [...]. J'ai quelques bons amis dans des pays lointains à qui jenvoie parfois des cadeaux. Ce mois-ci, j’ai acheté deux livres français, que jai expédiés par la poste, par voie de terre, à un Français. Or, quand je me suis présenté au bureau de poste, l'employée m’a demandé si je voulais expédier mes deux colis (deux livres) «par avion ou par... surface». Ce "par surface» est un détestable calque de l'anglais «surface mail». Je lui ai répondu que je préférais les envoyer "par voie terrestre». J’ai continué à parler et à répéter "par voie terrestre», mais elle a continué à utiliser son anglicisme "par surface». Je n'ai plus insisté, car je la connais bien; peut-être une irrécupérable. En commençant à remplir la déclaration, elle a écrit «One boo...». J’ai allongé le cou, j’ai lu, et c'en était trop! Je lui ai dit clairement quelle devait écrire «Un livre», et elle a recommencé. [...]. Pouvons-nous imaginer qu'une employée francophone d'un bureau de poste dans une région francophone ne sache pas utiliser les expressions "par voie terrestre», "par voie maritime» ou "par voie aérienne», selon le cas, et qu'elle s'obstine à dire l'anglicisme "par surface»? [...]. À mon âge, j’ai encore assez dénergie pour continuer à défendre ma langue et refuser qu'une employée des postes me serve des anglicismes dommageables. La personne a pourtant à sa disposition tout 
le matériel écrit et le vocabulaire français spécifique aux postes, mais elle préfère toujours utiliser l'anglicisme. La piètre qualité du français de cette employée de Postes Canada donne beaucoup à réfléchir. (LANDRY, 2019).

En Acadie, où le français est minoritaire, les formes linguistiques empruntées à la langue majoritaire, l'anglais, sont vues comme un danger. Le simple choix d'utiliser un emprunt constituerait alors une étape risquée vers l'assimilation linguistique. Dans la même ligne de pensée, certains scripteurs en viennent alors à voir ces pratiques linguistiques comme la marque d'un manque de fierté ou d'une absence de volonté de préserver l'identité collective (ARRIGHI ; VIOLETTE, 2013).

Ainsi, le chiac, tenu pour un parler hybride, est particulièrement condamné. Son utilisation, notamment par des artistes rencontrant un certain succès hors de frontières de l'Acadie (comme c'est le cas de la chanteuse country rock Lisa LeBlanc ou du groupe rap Radio Radio), est dénoncée:

Quand on n'a pas eu la chance de s'instruire, il est normal de ne pas savoir parler le français à un niveau du standard canadien, mais quand ce n'est pas le cas et qu'on détient un diplôme du secondaire et même universitaire et au nom d'une fausse fierté acadienne on choisit, avec ou sans guitare à la main, de s'exprimer en chiac, on fait erreur. On freine l'avancement d'une partie importante de l'Acadie des Maritimes. (LEBLANC, 2016. C'est nous qui soulignons)

Bien que constituant un écart à la norme, les archaïsmes, c'est-à-dire les formes linguistiques héritées de l'histoire du français (et ayant disparu 
d'autres régions francophones) sont souvent vus plus positivement ${ }^{8}$. Parce qu'ils renvoient à l'histoire de la langue et de l'établissement de l'Acadie, leur emploi est pris comme un gage de fidélité de la population acadienne à son passé, à ses valeurs et à ses traditions. Parce que certains de ces archaïsmes sont une marque de différenciation par rapport au Québec, ils sont aussi brandis comme l'indice de l'histoire et de l'identité distinctes du groupe acadien.

Le traitement différent des archaïsmes et des emprunts se donne dès lors à voir dans le discours de certains intervenants qui insistent sur la différence entre le français acadien traditionnel, une variété vue comme légitime, et le chiac si critiqué. On met aussi de l'avant que le chiac n'est pas la langue de tous les Acadiens mais seulement de ceux qui vivent à proximité des anglophones.

Le chiac n'est pas la langue acadienne. C'est un franglais parlé par les sympathiques acadiens de la région de Moncton ou du sud-ouest [sic] du Nouveau-Brunswick. La langue acadienne est une langue qui se distingue avec un bel accent, une diction claire et l'usage du vieux français [...]. Dans ma famille, on honore la langue française tout en étant bilingues quand il le faut. N'oubliez pas que c'est en raison de leur allégeance à la France, que les Acadiens ont été déportés. Un génocide. (LEBLANC, 2016)

Ces fréquentes condamnations publiques du vernaculaire se comprennent bien dans un terrain où les acteurs sociaux considèrent que c'est l'avenir et la vitalité de la communauté qui est en jeu (LEBLANC ; BOUDREAU, 2016). Puisque l'Acadie a lutté pour ses droits au nom d'une

8 Ces formes ont fait l'objet, dès la fin du $19^{\mathrm{e}}$ siècle, d'une défense et illustration par quelques érudits acadiens, dont Pascal Poirier, tenu pour le premier linguiste acadien (POIRIER, 1993). 
identité et d'une histoire distinctes, la langue joue un rôle majeur dans la construction de l'identité collective. Elle est en outre un des éléments qui légitime les aspirations et revendications politiques et sociales du groupe. Le mélange des langues, et plus encore, l'utilisation de l'anglais, en viennent alors à incarner, selon cette lecture, l'ultime menace pour la pérennité de la communauté francophone (ARRIGHI; VIOLETTE, 2013; ARRIGHI; URBAIN, 2019).

Ces débats renvoient également à deux idéologies hégémoniques dans la francophonie en général. On valorise à la fois une forme homogène de la langue, le français dit standard, comme étant un idéal pour la cohésion social et, pour les mêmes raisons, on met de l'avant l'importance du monolinguisme: tout écart, interne ou externe, est dès lors dévalorisé. Ces deux idéologies, l'idéologie du standard (MILROY ; MILROY, 2001) et l'idéologie du monolinguisme (BLACKLEDGE, 2000 ; KLINKENBERG, 2001), centrales dans la majorité des pays occidentaux depuis l'avènement des État-nations, soutiennent ainsi qu'une langue commune, unique et homogène est non seulement désirable mais plus encore nécessaire pour vivre en société (CANUT ; DUCHENE, 2011). Les communautés francophones minoritaires canadiennes reproduisent souvent, en dépit du multiculturalisme et du bilinguisme officiel, ces conceptions monolingues, comme par exemple lorsqu'elles plaident pour des institutions homogènes francophones et, au Nouveau-Brunswick, le modèle de la dualité linguistique (dont nous avons traité plus haut; voir aussi Heller, 2007). Dans ce cadre idéologique, chaque membre de la communauté minoritaire se voit attribuer une responsabilité citoyenne de préservation de la langue: sécarter de la norme est alors conçu comme du laxisme, de l'indifférence voire un manque de respect par rapport à l'avenir collectif (plus haut un participant au débat parle d' « inconscience linguistique ») :

Un fait demeure : le chiac est un dialecte [...] à 25 
kilomètres et plus à l'extérieur de cette région qu'est Moncton-Dieppe, peu de personnes ne le comprennent [sic]. Ce qui se parle aujourd'hui, c'est du franglais ou plutôt de l'anglais avec un peu de français ! [...] Si nous acceptons et valorisons cette situation, serions-nous prêt [sic] à accepter que les mathématiques, l'histoire, etc.., soit présentés avec des réponses approximatives ? Eh bien non ! Alors pourquoi accepter de bafouer le français, cette langue qui nous définit? Nous n'avons quà lire les propos haineux qui paraissent sur le site $\mathrm{CBC}$ pour réaliser le dommage que cela cause.

(ALLARD, 2016. C'est nous qui soulignons)

Toutefois, notre corpus montre que le rapport au standard reste ambigu. Alors qu'au Québec, province où le français est majoritaire, l'élaboration d'une langue standard québécoise a été institutionnalisée par l'Office de la langue française et diffusée dans la population (BIGOT, 2008), l'Acadie, plus aux marges, n'a pas pu se doter d'une norme linguistique distincte. À ce titre, les formes régionales ne sont pas considérées comme "de bon aloi " dans des contextes formels. Bien que les Acadiens et Acadiennes utilisent une variété de registres de langue (du plus familier et au plus formel), les débats dans les médias caricaturent souvent les pratiques linguistiques en les réduisant aux formes les plus vernaculaires. Dans les discours sur la langue, il est donc rare d'intégrer une vision nuancée de la valeur et de l'utilité de la variation linguistique (BERUBE, 2010 en fait une très bonne analyse en contexte scolaire).

Dans ce contexte, seuls certains domaines sont ouverts à la valorisation des pratiques régionales. Les arts sont l'un de ces domaines où est permise une certaine licence poétique (LECLERC, 2016) en autant qu'elle reste bien circonscrite. À côté de ce marché franc, le marché officiel (pour reprendre la terminologie de Bourdieu, 1977, 1982) ne tolère guère d'entorses à ce qui est jugé comme la bonne langue. L'institution scolaire est 
ainsi perçue comme un milieu où la variété n’est pas appropriée (LANDRY, 2012) et où l'on idéalise une langue standard souvent mythifiée. De même à l'Université, ces rapports de force entre pratiques vernaculaires et langue standard sont reproduits, comme l'ont montré les travaux de Vernet (2017). Dans sa recherche, il a fait du terrain dans les cours mis en place pour apprendre à écrire et à parler en français standard aux étudiants et étudiantes de l'Université de Moncton. Il note que, dans ces cours

« [1]e « standard » [...] une variété imaginée et abstraite, perçue comme valorisante et valorisée en société, et comme globalement stable; [reste] impossible à définir dans ses formes car il s'agit d'une idéalisation plus que d'une variété observable. [...] [ainsi] aucun des critères cités [par les enseignants et enseignantes] ne permet de se faire une idée claire de « ce qu'est le standard » et, bien souvent, il sera décrit en négatif, on dira plutôt ce que ce n'est pas. » (VERNET, 2017, p. 185)

La sacralisation du standard en milieu éducatif amène parfois à des positionnements extrêmes où la variation est condamnée même lorsqu'elle est utilisée dans des fonctions ludiques et pour être fidèle à la réalité locale. Un cas exemplaire l'illustre parfaitement: celui des débats entourant le film 2 faces. Ce film réalisé par des élèves d'une école secondaire de la région du grand Moncton et par un cinéaste local, Chris LeBlanc, visant à lutter contre l'intimidation en milieu scolaire et tourné dans le vernaculaire des élèves, a créé scandale et s'est fait interdire par le district scolaire (le commanditaire du film). Bien que le chiac y soit utilisé dans un contexte artistique (où les jeunes acteurs et actrices participent à des d'échanges qui se veulent informels), la diffusion du film en contexte scolaire et l'interdiction de sa diffusion a remis sur la table les débats autour de la valeur de ce parler et de 
sa légitimité. Cet exemple illustre bien que dans un marché officiel comme celui de l'école, en dépit de sa valeur identitaire, le vernaculaire est encore condamné au profit de la langue standard.

En réaction aux critiques du vernaculaire, de plus rares interventions, prises de parole de jeunes militants ou de linguistes, essaient de nuancer le propos et se portent à la défense du parler acadien, que ce soit dans ses formes traditionnelles ou dans ses formes les plus contemporaines notamment hybrides. Dans ces plaidoyers, les usages locaux sont valorisés pour leur portée identitaire et leur authenticité9.

Un bon exemple de ces discours de défense s'est donné à voir lorsque des citoyens ont choisi de défendre publiquement le slogan choisi pour les Jeux de la francophonie canadienne, Right fier (voir extrait ci-dessous). Ils ont souligné quà force de critiquer le vernaculaire des jeunes, le discours public en venait à créer ou renforcer l'insécurité linguistique, ultimement dangereuse pour la vitalité de la communauté. Au lieu d'envisager l'expression chiaque "right fier " comme une marque d'assimilation, ils proposent d'y voir le signe d'une utilisation ludique et décomplexée de la langue :

Le slogan Right fiers semblait s'imposer de soi pour illustrer le dynamisme linguistique et le caractère unique de nos villes hôtes, estime Alec Boudreau, président de la FJCF. C'est à la fois un clin d'oil au chiac acadien, et une ouverture à la Francophonie sous toutes ses formes et avec tous ses accents (DELATTRE, 2016).

Dans certains articles, toutefois moins fréquents que ceux qui

9 Notons que cette équation-là est strictement repoussée par d'autres, ainsi le chroniqueur du nord-ouest de la province Rino Morin Rossignol affirme de façon imagée que « le chiac [...] est à lâme acadienne ce que l’athéisme est à la foi chrétienne. " (Rino Morin Rossignol, " Une fête de la liberté ", L’Acadie Nouvelle, 4 mai 2018) 
critiquent le chiac, on souligne donc sa valeur communautaire. Cette variété permet de marquer l'ancrage dans une région et devient le symbole de l'identité collective. Elle joue ainsi à sa façon le rôle d'une norme, norme locale et " communautaire » cette fois - ce que le sociologue Gumperz (1982) nomme le we-code, qui permet de marquer l'appartenance au groupe. C'est précisément cela que fait valoir un participant au débat:

Léducation permet aux Francophones d'ici et d'ailleurs d'apprendre cette variété de français qui est notre patrimoine commun, [...]. Mais elles [les écoles] doivent aussi inculquer la notion que le chiac, le ch'ti et le shawiniganais ont leur propre utilité et leur propre valeur. On parle ces variétés de français justement parce que nous sommes des "personnes normales" qui avons ce désir profond de communion avec nos pairs et de communiquer avec eux dans le registre qui nous unit. Il n'y a pas de honte à être une personne normale; il n'y pas de honte à parler " comme on parle chez nous ", que l'on vienne d'Edmundston, de Winnipeg, ou d'Aix-en-Provence. (POLIQUIN, 2018)

Cette valeur identitaire et communautaire, cette marque d'appartenance locale et régionale, sont aussi ce qui amène à défendre d'autres spécificités linguistiques acadiennes (moins menaçantes pour l'identité du groupe que le mélange des langues), comme les archaïsmes ou les mots régionaux par exemple.

\section{Conclusion: un rapport ambivalent à la norme linguistique}

Notre analyse des discours médiatiques sur la qualité de la langue en Acadie publiés ces cinq dernières années montre bien que derrière un 
discours sur les formes linguistiques se cache toujours des enjeux sociaux et politiques bien plus larges. Si ce sont les façons de parler qui sont en ligne de mire, elles ne sont que le prétexte pour actualiser toute une série d'idéologies linguistiques et nationales profondément ancrées en Acadie et dans la francophonie. Les points de vue divergents sur ce qui constitue un français acceptable et désirable renvoient surtout à des visions concurrentes de la communauté, ses valeurs et son avenir.

Parce que la langue minoritaire en vient à incarner l'identité distincte du groupe, elle a historiquement servi d'élément central des revendications sociales et politiques. Dans un tel contexte, les discussions sur sa nature, sa définition, ses limites abondent. Plusieurs normes en concurrence coexistent. Certains privilégient le purisme linguistique en voyant dans le prestige qui lui est associé un outil de légitimation fort, notamment au plan politique. Cela les amène à dévaloriser les formes régionales. D’autres défendent les pratiques régionales en raison de leur rôle symbolique et communautaire. Le parler acadien se voit doté d' "effets d'authenticité » (ECKERT, 2003), alors que l'évaluation des emprunts à l'anglais et du mélange des langues reste ambivalente (VIOLETTE, à paraitre). Comme on l'a vu, la valorisation du vernaculaire reste tributaire des contextes dans lesquels on l'utilise. Ces dernières années, si le monde artistique, par exemple, a accordé plus de place à la variation régionale (LECLERC, 2016; BOUDREAU, 2016), cette ouverture reste toutefois restreinte. Comme dans les milieux majoritaires, le français oral, ordinaire et régional reste souvent dévalorisé dans les marchés officiels et ne sépanouit vraiment que dans les marchés francs.

\section{Références}

ALLARD, Marcel. Accepter de bafouer la langue française? Mon Opinion, L'Acadie Nouvelle, 21 mars, 2016. https://www.acadienouvelle.com/mon- 
-opinion/

ARRIGHI, Laurence; URBAIN, Émile. Les « mauvais exemples » de la francophonie nord-américaine: intérêt des acteurs, construction des images, instrumentalisation des groupes. In: HALLION, Sandrine; ROSEN, Nicole (org.) avec NAJAT, Sandra (coll.) Les français d'ici: des discours et des usages. Québec: Presses de l'Université Laval, 2019. p. 11-32.

- Wake up Québec : du recours aux communautés francophones minoritaires dans le discours visant l'émancipation nationale du Québec. Francophonies d'Amérique, n. 42-43, p. 107-126, 2016/2017.

Arguments et enjeux d'une instrumentalisation linguistique Lexemple du rapprochement Acadie-Louisiane, Beatrice Bagola et Ingrid Neumann-Holzschuh. L'Amérique francophone - Carrefour culturel et linguistique, Francfort: Peter Lang Verlag, p. 163-189, 2016.

ARRIGHI, Laurence; Isabelle VIOLETTE. De la préservation linguistique et nationale : la qualité de la langue de la jeunesse acadienne, un débat linguistique idéologique, Revue de l'Université de Moncton, v. 44, n. 2, p. 67-101, 2013.

. Le discours sur la qualité de la langue au Nouveau-Brunswick. In: LANDRY, Michelle; MASSICOTTE, Julien; PÉPIN-FILLION, Dominique (dir.). L'État de l'Acadie. Del Busso: Montréal. À paraitre.

ARRIGHI, Laurence; BERGER,Tommy. Le chiac dans les médias sociaux. Entre spontanéité, créativité et réflexivité linguistiques. Revue transatlantique d’études suisses. À paraitre.

BÉRUBÉ, Julie. Discours et idéologies linguistiques en Acadie du Nouveau-Brunswick - Lexemple de la presse et du milieu scolaire. 2010.Thèse de doctorat. Université de Moncton. 2010.

BIGOT, Davy. « Le point » sur la norme grammaticale du français québécois 
oral.2008. Thèse de doctorat. Université du Québec. 2008.

BLACKLEDGE, Adrian. Literacy, power and social justice. Stoke-onTrent:Trentham Books. 2000.

BLOMMAERT, Jan. Language Ideological Debates. La Haye: Mouton De Gruyter. 1999.

. Discourse. A Critical Introduction. Cambridge: Cambridge University Press. 2005.

BOUCHARD, Chantal. Méchante langue. La légitimité linguistique du français parlé au Québec. Presses de l'Université de Montréal: Montréal, 2011

. La langue et le nombril - Une histoire sociolinguistique du Québec. Fides, Montréal, 2002.

BOUDREAU, Annette. À l'ombre de la langue légitime: l'Acadie dans la francophonie, Paris: Classiques Garnier, 2016.

. Des voix qui se répondent: analyse discursive et historique des idéologies linguistiques en Acadie: l'exemple de Moncton. Minorités linguistiques et société, n. 4, p. 175199. https://doi.org/10.7202/1024697ar.

. La construction des représentations linguistiques: le cas de l'Acadie. Revue Canadienne de Linguistique, v. 54, n. 3, p. 439-459, 2009.

BOUDREAU, Annette ; URBAIN, Émilie. La presse comme tribune d'un discours d’autorité sur la langue: représentations et idéologies linguistiques dans la presse acadienne, de la fondation du Moniteur Acadien aux Conventions nationales. Francophonies d'Amérique, n. 35, p. 23-46, 2013.

BOURDIEU, Pierre. Ce que parler veut dire. Léconomie des échanges linguistiques. Paris, Fayard. 1982. 
. Léconomie des échanges linguistiques. Langue française, $\mathrm{n}^{\circ} 34, \mathrm{p}$. 17-34, 1977.

BOURQUE, Denis ; RICHARD, Chantal. Les Conventions nationales acadiennes. Tome I : 1881-1890. Moncton: Institut d'études acadiennes. 2014.

CAMERON, Deborah. Verbal Hygiene. Londres: Routledge. 1997.

CANDEA, Maria; VÉRON, Laélia. Le français est à nous! Petit manuel d'émancipation linguistique. Paris: La découverte, 2019.

CANUT, Cécile ; DUCHÊNE, Alexandre. Introduction. Instrumentalisations politiques et économiques des langues: le plurilinguisme en question, Langage \& société, n. 136, p. 5-12, 2011.

CORMIER, H. La langue, un projet de société, peut-être ?, Mon Opinion, L'Acadie Nouvelle, 12 mars 2018. https://www.acadienouvelle.com/mon-opinion/

CROTEAU, Jean-Philippe ; MARTINEAU, France ; FRENETTE, Yves. La représentation de la notion et de la langue dans la presse canadienne-française, 1912-1913. In : ARRIGHI, Laurence ; GAUVIN, Karine (dir.) Regards croisés sur les français d'ici. Québec: Presses de l'Université Laval, p. 179-198. 2018.

DAIGLE, Jean. LAAcadie de 1604 à 1763, synthèse historique. In : DAIGLE, Jean (dir.). L’Acadie des maritimes. Moncton: Institut d'études acadiennes, p. 3-43. 1993.

DELATTRE, Simon. Right fiers, le slogan des Jeux de la Francophonie dérange, Reportage, L'Acadie Nouvelle, 5 février 2016. https://www.acadienouvelle.com/actualites/2016/02/04/right-fiers-le-slogan-des-jeux-de-lafrancophonie-canadienne-derange/

DUGUAY, Benoît. Des idées sur l'«insécurité», Mon opinion, L'Acadie Nouvelle, 5 mars 2018. https://www.acadienouvelle.com/mon-opinion/ 
. L'Acadie mérite mieux, Mon opinion, L'Acadie Nouvelle, 23 aout 2019. https://www.acadienouvelle.com/mon-opinion/

ECKERT, Penelope. Sociolinguistics and authenticity: An elephant in the room, Journal of Sociolinguistics, vol. 7, no 3, p. 392-397. 2003.

FRANCARD, Michel. Le français d'ici est-il du français ? La construction des jugements de normativité dans les communautés francophones périphériques européennes. In : ARRIGHI, Laurence ; GAUVIN, Karine (dir.). Regards croisés sur les français d'ici. Québec : Les presses de l'Université Laval. Collection Les voies du français, pp. 251-268. 2018.

. L'insécurité linguistique en Communauté française de Belgique. Bruxelles: Service de la langue française, 1993.

FORGUES, Éric, ROBINEU, Anne; PÉPIN-FILION, Dominique; BOUCHARD, Marc-André. La construction d'espaces francophones comme projet de société en milieu minoritaire. Minorités linguistiques et société / Linguistic Minorities and Society, n.13, p. 26-45, 2020. https://doi. org/10.7202/1070389ar

GEE James Paul. An introduction to Discourse Analysis. Theory and Method. Londres: Routledge. 1999.

GUEUNIER, Nicole; GENOUVRIER, Émile; KHOMSI, Abdelhamid. Les Français devant la norme. Contribution à une étude de la norme du français parlé. Paris: Champion. 1978. GUMPERZ, John. Discourse Strategies. Cambridge: Cambridge University Press. 1982.

HALLION, Sandrine; MONNIN, Isabelle. M. Dulong fait-il une enquête ou une autopsie? Un discours expert comme catalyseur dans la circulation d'idéologies linguistiques au Manitoba (1963-1964). Francophonies d’Amérique, n. 42-43. p. 69-104. 2016-2017 
HELLER, Monica. Paths to Postnationalism: A Critical Ethnography of Language and Identity. Oxford: Oxford University Press. 2011.

. Media, the State and Linguistic Authority. In: Johnson, Sally; MILANI, Thomasso. Language ideologies and media discourse: Texts, practices, politics. Londres: Continuum, p. 277-282. 2010.

- "Langue ", " communauté » et " identité »: le discours expert et la question du français au Canada, Anthropologie et Sociétés, v. 31 n. 1, p. 39-54. 2007.

. Éléments d'une sociolinguistique critique. Paris: Didier. 2002.

JOHNSON, Sally; MILANI, Thomasso. Language ideologies and media discourse: Texts, practices, politics. Londres: Continuum. 2010.

KLINKENBERG, Jean-Marie. La langue et le citoyen. Pour une autre politique de la langue française. Paris: PUF, 2001.

KROSKRITY, Paul V. Regimes of Language: Ideologies, Polities, and Identities. Santa Fe: School of American Research Press. 2000.

LANDRY, Jonathan. Étude de représentations linguistiques de jeunes Acadiennes et Acadiens en milieu scolaire: vers un éveil à sa propre langue? Thèse de maitrise - Université de Monct, 2012.

LANDRY, Merville. Je suis francophone, Mon Opinion, L'Acadie Nouvelle, 30 aout 2019. https://www.acadienouvelle.com/mon-opinion/

LEBLANC, Anne Évangéline. Honorer la langue française et acadienne, Mon Opinion, L’Acadie Nouvelle, 4 novembre 2016. https://www.acadienouvelle.com/mon-opinion/

LEBLANC, Dollard. Combattre notre complexe d'infériorité, Mon Opinion, L’Acadie Nouvelle, 15 avril 2016. https://www.acadienouvelle.com/ 


\section{mon-opinion/2016/04/14/combattre-complexe-dinferiorite/}

LEBLANC, P. L'Acadie mérite mieux, Mon opinion, L'Acadie Nouvelle, 30 aout 2019. https://www.acadienouvelle.com/mon-opinion/2019/08/29/ lacadie-merite-mieux/

LECLERC, Catherine. Radio Radio à Montréal: "la right side of the wrong". Revue de l'Université de Moncton, v. 47, n.2, p. 95-128, 2016.

LEDEGEN, Gudrun. Le bon français: Les étudiants et la norme linguistique. Paris: L'Harmattan. 2000.

MILROY, James; MILROY, Lesley. Authority in Language: investigating standard English. Oxford: Routledge, 2001.

PAVEAU, Marie-Anne; ROSIE, Laurence. La langue française, passions et polémiques. Paris: Vuibert. 2008.

PERROT, Marie-Ève. Aspects fondamentaux du métissage français / anglais dans le chiac de Moncton (Nouveau-Brunswick, Canada). 1995.Thèse de doctorat - Université Paris 3.

POIRIER, Pascal. Le glossaire acadien. Moncton, Centre d'études acadiennes, 1993.

POLIQUIN, Gabriel. Le français de Shédiac vaut bien le français parisien, Forum public, L'Acadie Nouvelle, 15 février 2018. https://www.acadienouvelle.com/

REMYSEN, Wim. Les représentations identitaires dans le discours normatif des chroniqueurs de langage canadiens-français depuis le milieu du XIXe siècle. Journal of French Language Studies, v. 22, n. 3, p. 419-445, 2012. 
RENAULT, J. Dieppe: I don't speak », Mon opinion, L'Acadie Nouvelle, 8 novembre 2018.

ROBILLARD, Didier. (de) Peut-on construire des "faits linguistiques " comme chaotiques? Éléments de réflexion pour amorcer le débat. Marges linguistiques, n. 1, p. 163-204, 2001.

ROSSIGNOL, Rino Morin. Assimilons-nous!. L'A cadie Nouvelle, chroniques. 22 mars, 2016. https://www.acadienouvelle.com/chroniques/2016/03/22/ assimilons-nous/

URBAIN, Émilie. Histoire de la construction d'un discours métalinguistique d'autorité. Une étude des débats langagiers idéologiques dans la presse francophone louisianaise depuis la Guerre de Sécession. 2014. Thèse de doctorat Université de Liège et Université de Moncton. 2014.

VERNET, Samuel. Discours, idéologies linguistiques et enseignement du français à l'Université de Moncton, en Acadie du Nouveau-Brunswick. Études canadiennes / Canadian studies, n. 83, p. 177-194, 2017.

VIOLETTE, Isabelle. " Parler chiac, c'est même pas fake »: immigration, authenticité et français vernaculaire en Acadie du Nouveau-Brunswick (Canada). In : URBAIN, Émilie; ARRIGHI, Laurence. Penser les langues et la sociolinguistique à partir des marges. Québec: Presses de l'Université Laval. À paraitre.

. Immigration francophone en Acadie du Nouveau-Brunswick. 2010. Thèse de doctorat - Université de Moncton et Université de Tours.

Debates sobre a norma e a qualidade da língua em meio minoritário: o exemplo da Acádia do Nova-Brunswick

Resumo: Com base nos discursos publicados na principal mídia francófona da região nos últimos cinco anos, nós analisamos os discursos sobre a língua 
francesa que circulam em uma comunidade minoritária francófona no Canadá, a Acádia da Nova Brunswick. O objetivo do nosso artigo é fornecer um retrato dos debates sobre a qualidade do francês e a norma linguística que estão surgindo nesta região caracterizada pelo uso generalizado de vernáculos marcados pela variação linguística (seja em termos de arcaísmos, variação morfossintática ou contato entre as línguas). Em um contexto minoritário em que os contatos linguísticos com a língua dominante, o inglês, são numerosos e multifacetados, o discurso sobre a qualidade do francês falado (ou escrito) na região é politicamente carregado e está ligado a questões de nacionalismo, de representação política e de direito. Nosso estudo mostra, assim, como comentários sobre a linguagem, sejam feitos por atores sociais comuns sejam por figuras públicas, revelam questões sociais, políticas e econômicas mais amplas. De forma mais específica, nossas análises revelam que os discursos frequentemente críticos sobre o francês falado nessa região giram em torno de questões de acesso e compartilhamento de recursos: acesso ao mercado de trabalho, a serviços públicos, à Francofonia internacional e as oportunidades de desenvolvimento econômico que ela poderia favorecer.

Palavras-chave: Francês acadiano. Língua padrão. Minorias linguísticas. Canadá.

Abstract: Based on discourses published in the main Francophone media in the region over the past five years, we analyzed discourses about the French language circulating in a minority francophone community in Canada, the Acadia of New Brunswick. The goal of our article is to provide a portrait of the debates about the quality of French and the linguistic norm emerging in this region characterized by the widespread use of vernaculars marked by linguistic variation (whether in terms of archaisms, morphosyntactic variation or contact between languages). In a minority context in which the linguistic contacts with the dominant language, English, are numerous and multifaceted, the discourse on the quality of spoken (or written) French in the region is politically charged and linked to issues of nationalism, representation politics and rights. Thus, our study shows how comments on language, whether made by ordinary social actors or public figures, reveal broader social, political and economic issues. More specifically, our analyzes reveal that the often critical speeches about the French spoken in this region revolve around issues of access and resource sharing: access to the labor market, public services, international francophonie and the opportunities for economic development that it could favor.

Keywords: Acadian French. Standard language. Linguistic minorities. Canada.

\section{Recebido em: 16/02/2021}

\section{Aceito em: 20/04/2021}

